\title{
Valorisation des sédiments de dragage en technique routière, cas de la rivière de la Soummam dans la région de Bejaia au nord de l'Algérie
}

\author{
Brahim BANOUNE ${ }^{1}$, Bachir MELBOUCI ${ }^{2}$, Frédéric ROSQUOËT ${ }^{3}$, \\ Thierry LANGLET ${ }^{3}$
}

1. Université de Bejaia, Département génie civil, Bejaia, Algérie. brahim.banoune@gmail.com

2. Université de Tizi Ouzou, Département de génie Civil, Tizi Ouzou, Algérie. melbouciba@hotmail.fr

3. Laboratoire des Technologies Innovantes, Université de Picardie Jules Verne, France. frederic.rosquoet@u-picardie.fr,thierry.langlet@u-picardie.fr

\section{Résumé :}

Afin d'aborder l'emploi et la valorisation des sédiments de dragage et rentabiliser l'opération de dragage qui s'avère très onéreuse, nous avons étudié le cas des sédiments de l'Oued de la Soummam dans la région de Bejaia au nord d'Algérie.

Une étude paramétrique approfondie qui a pour objectif d'utiliser les sédiments de dragage dans le domaine routier a été réalisée. Elle a comme double avantage, d'une part de rentabiliser l'opération de dragage et d'avoir à disposition une grande quantité de matériaux de construction.

Comme première étape, nous avons étudié les caractéristiques minéralogiques et physiques des deux échantillons, discuté les performances mécaniques, pour ensuite aborder la possibilité d'amélioration des caractéristiques physico-mécaniques de ces sédiments, permettant ainsi leur utilisation. Cette pré-étude montre qu'il est nécessaire d'améliorer les caractéristiques physiques et le comportement mécanique des sédiments naturels pour répondre aux critères imposés par les réglementations en vigueur.

L'effet d'un traitement au ciment seul ou associé à des fines de carrière sur les propriétés des sédiments a été étudié. Différentes formulations sont testées dans le but de répondre aux exigences réglementaires.

Ainsi nous avons noté que la portance et la résistance à la compression des sédiments se sont nettement améliorées après traitement et évoluent avec le taux de ciment, comme nous avons noté un effet positif de l'association de fines de carrière sur les mêmes paramètres. Une synthèse des caractéristiques mécaniques obtenues est proposée. Le matériau traité est désormais conforme aux normes exigées en technique routière à partir de $2 \%$ de ciment.

\section{Mots-clés :}

Caractérisation des sédiments, Valorisation, Ciment, Techniques routières, Traitement de sédiments. 


\section{Thème 6 - Gestion durable des zones littorales et estuariennes}

\section{Introduction}

Le dragage des rivières est un défi environnemental et économique majeur, et la gestion des grandes masses de sédiments dragués devient une préoccupation croissante. Des techniques de stabilisation sont développées pour valoriser ces sédiments (BOUTOUIL, 1998 ; SEMCHA, 2006 ; SCORDIA, 2008 ; ZENTAR et al., 2009 ; REKIK \& BOUTOUIL, 2011; DUBOIS et al, 2009 ; SILITONGA, 2010; WANG, 2011 ; BANOUNE et al., 2016 ; MAROUF, 2018) comme alternative au rejet en mer ou au stockage. La matière passe alors de déchets simples à enlever à des ressources naturelles précieuses (LEVACHER et al, 2006; ANGER, 2014). Ces techniques s'avèrent également plus écologiques.

Cependant, il est clair que toute décision de traitement et de récupération dépend des résultats des analyses. Les sédiments ciblés dans cette étude proviennent de la rivière Soummam, dans la région de Bejaia, au nord de l'Algérie.

Une caractérisation physique, minéralogique et mécanique a été réalisée, puis discutée pour l'utilisation de ces sédiments dans le secteur routier, considéré comme l'un des secteurs qui consomment le plus de granulats. Cette première étude a révélé la prédominance de particules fines avec un faible pourcentage d'argile et une présence de matières organiques.

Le traitement de ces sédiments avec des liants est nécessaire pour améliorer les caractéristiques physiques et mécaniques des sédiments naturels et envisager leur valorisation en couche de forme dans les structures de chaussée, conformément à la réglementation en vigueur GTR (LCPC et SETRA, 2000a). Pour cela, une stabilisation à base de ciment seul ou avec des additifs tels que des fines de carrière a été réalisée en faisant varier le taux de ciment dans les sédiments dans un premier temps, puis en associant des fines de carrière dans un second temps. Le taux de ciment est variable dans des rapports allant de 2 à $10 \%$ en matière sèche (sédiments +ciment). Ensuite nous avons examiné l'effet de l'ajout des fines de carrière à des pourcentages variés pour $2 \%$ de ciment seulement.

Les résultats montrent une amélioration substantielle des caractéristiques mécaniques des sédiments traités permettant ainsi leur valorisation comme couche de forme de chaussée en respect de la réglementation en vigueur.

\section{Matériaux et méthodologie}

\subsection{Caractéristiques des sédiments}

Les sédiments étudiés sont prélevés à l'embouchure du l'Oued de la Soummam situé à Bejaia au Nord d'Algérie. La nature des sédiments récupérés et la connaissance de leurs caractéristiques physiques et mécaniques sont indispensables pour discuter de leur valorisation à l'état naturel ou après traitement. 


\section{XVIèmes Journées Nationales Génie Côtier - Génie Civil \\ Le Havre 2020}

Les caractéristiques des sédiments et la méthodologie de leur traitement été étudié précédemment (BANOUNE et al., 2016). Une prédominance du quartz et de la dolomite lors de l'étude minéralogique à travers la diffraction aux rayons $\mathrm{X}$ a été notée, voir tableau 1.

Tableau 1. Composition minéralogique des sédiments.

\begin{tabular}{llll}
\hline Sédiments & \multicolumn{2}{l}{ Composition minérale (\%) } & Principaux minéraux \\
\hline & Quartz & Dolomite & \\
\cline { 2 - 4 } Soummam $(S)$ & $55-60$ & $40-45$ & Quartz, Dolomite \\
\hline
\end{tabular}

La fraction fine qui a un diamètre $<80 \mu$ m représente plus $80 \%$ des particules comme montré sur la figure 1. L'argilosité des sédiments est aussi un paramètre d'une importance majeure pour le choix du traitement à apporter à notre matériau.

L'indice de plasticité IP et la valeur au bleu de méthylène VBS renseignent sur l'argilosité des sédiments GTS (LCPC et SETRA, 2000b). L'indice de plasticité IP, déterminé à l'aide des limites d'Atterberg selon la norme française NF P94-051 (AFNOR, 1993a). Les sédiments sont alors classés comme des sols peu plastiques selon Casagrande. L'essai au bleu de méthylène confirme ce résultat (Tableau 2). La distribution granulométrique révèle une texture limoneuse avec une faible proportion de particules fines $(<2 \mu \mathrm{m})$. Le calcul des coefficients d'uniformité $(\mathrm{Cu}>2)$ et de courbure $\mathrm{Cc}(1<\mathrm{Cc}<3)$ révèle une granulométrie étalée.

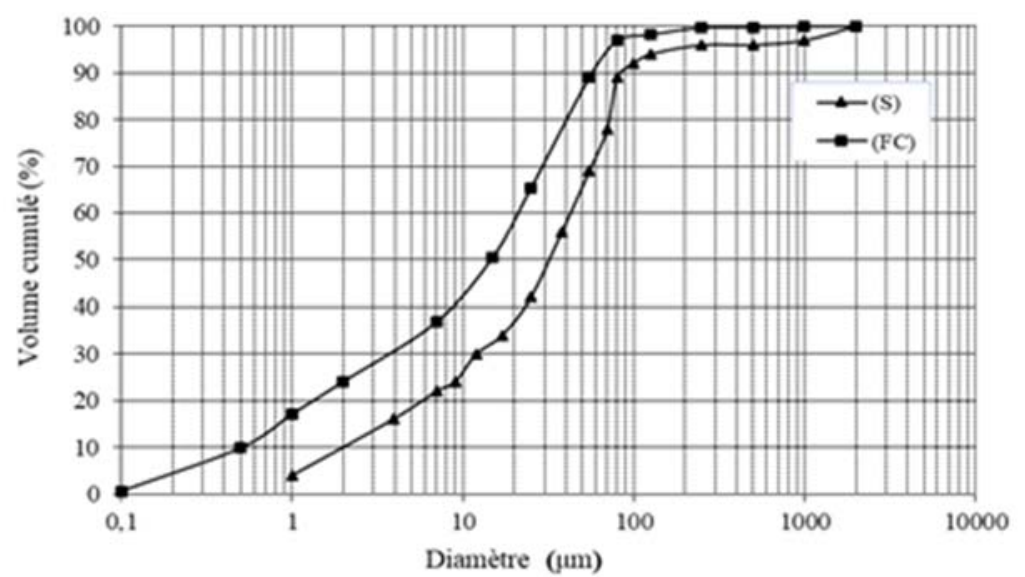

Figure 1. Courbes granulométriques, (S) : Sédiments de la Soummam, FC : Fines de carrière.

Les principales caractéristiques mesurées sont récapitulées dans le tableau 2.

Le comportement des sols est modifié considérablement et leur stabilité volumique dans le temps est mise en cause par la présence de matières organiques en quantité non négligeable (MAHERZI \& BEN ABDELGHANI, 2014). 


\section{Thème 6 - Gestion durable des zones littorales et estuariennes}

Tableau 2. Caractéristiques des sédiments.

\begin{tabular}{ll}
\hline Paramètres & Sédiments de la Soummam (S) \\
\hline$\%<2 \mu \mathrm{m}$ (Argile) & $10 \%$ \\
$2 \mu \mathrm{m}<\%<63 \mu \mathrm{m}$ (Limon) & $61 \%$ \\
$\%>63 \mu \mathrm{m}$ (Sable fin) & $29 \%$ \\
$\%<80 \mu \mathrm{m}$ & $85 \%$ \\
$\rho_{\mathrm{s}}\left(\mathrm{g} / \mathrm{cm}^{3}\right)$ & 2,74 \\
Coefficient d'uniformité Cu & 22 \\
Coefficient de courbure Cc & 1,64 \\
VBS & 2,55 \\
MO par pertes au feu (\%) & 3,90 \\
$W_{l}(\%)$ & 41,50 \\
$W_{p}(\%)$ & 26,40 \\
IP (\%) & 15,10 \\
Principaux minéraux & Quartz, Dolomite \\
\hline
\end{tabular}

\subsection{Caractéristique mécanique}

La caractérisation mécanique du matériau au compactage est une étape d'égale importance que la caractérisation physique. En effet, elle nous permet de classifier le matériau selon le Guide des Terrassements Routiers (LCPC and SETRA, 2000a). Les sédiments que nous étudions font partie de la classe $\mathrm{F}_{11} \mathrm{~A}_{2}$ qui regroupe les sols limoneux peu plastiques à faible taux d'argile. La classe obtenue favorise l'utilisation de ce matériau dans le domaine de la géotechnique. Toutefois, la détermination des paramètres de compactage est nécessaire, afin de mieux cerner les conditions de leur mise en œuvre. Les indices de portance immédiats (IPI) et les indices CBR après immersion $\left(I_{\mathrm{CBR}}\right)$ des sédiments avant traitement sont déterminés. Ils sont obtenus par poinçonnement selon la norme française NF P94-078 (AFNOR, 1997) sur des éprouvettes confectionnées à l'essai Proctor normal (AFNOR, 1999). Les résultats obtenus dans le tableau 3 sont inférieurs aux normes exigées dans le domaine routier (LCPC et SETRA, 2000a), une utilisation en l'état de ces sédiments dans le domaine des travaux routiers est non conforme. Les sédiments doivent être stabilisés par ajout de liant hydraulique tel que le ciment.

\section{Résultats et discussion}

Nous avons fait varier les teneurs en ciment $(\mathrm{C})$ entre $2 \%$ et $10 \%$ de la matière sèche. Ensuite nous avons fait varier la teneur en fines de carrière entre 5 et $25 \%$ tout en fixant la teneur en ciment à $2 \%$. L'apport du traitement sera jugé à travers la variation de la résistance à la compression simple $(\mathrm{Rc})$ et de la portance. Les paramètres de compactage ( $W_{\text {opt }}$ et $\gamma_{\text {dopt }}$ ) sont déterminés pour chaque formulation à l'énergie Proctor normal.

Le traitement au ciment a permis d'accroitre les performances mécaniques. Ceci est constaté dans le tableau 3 à travers l'évolution l'indice de portance immédiat (IPI) et 


\section{XVIèmes Journées Nationales Génie Côtier - Génie Civil \\ Le Havre 2020}

l'indice $\mathrm{CBR}$ après immersion $\left(I_{\mathrm{CBR}}\right)$. Notons également une nette amélioration du rapport ( $\left.I_{C B R} / \mathrm{IPI}\right)$ en fonction du dosage en ciment. Ceci témoigne de l'efficacité du traitement du point de vue de la portance. Ainsi, non seulement le risque de perte de cohésion en cas de saturation hydrique de la couche est écarté, mais les rapports (ICBR/IPI) sont tous nettement supérieurs à 1 , ce qui est un critère de pérennité des traitements envisagés (LCPC et SETRA, 2000b).

Le deuxième paramètre, la résistance en compression simple Rc, est étudié sur des éprouvettes cylindriques d'élancement $2(5 \times 10 \mathrm{~cm})$, confectionnées à l'optimum Proctor, selon la norme française NF P98-230-2 (AFNOR, 1993b). Elles sont conservées dans les conditions d'une cure normale à l'air libre à la température du laboratoire $20 \pm 2^{\circ} \mathrm{C}$, et sont testées à 7, 14, 21, 28, 60 et 90 jours.

Tableau 3. Caractéristiques mécaniques des sédiments en fonction du taux de ciment.

\begin{tabular}{lrrrrrr}
\hline Dosage en ciment (\%) & \multicolumn{1}{c}{$\boldsymbol{0}$} & \multicolumn{1}{c}{$\mathbf{2}$} & \multicolumn{1}{c}{$\mathbf{4}$} & \multicolumn{1}{c}{$\boldsymbol{6}$} & \multicolumn{1}{c}{$\boldsymbol{8}$} & \multicolumn{1}{c}{$\mathbf{1 0}$} \\
\hline$\gamma_{\text {d opt }}\left(\mathrm{g} / \mathrm{cm}^{3}\right)$ & 1,79 & 1,76 & 1,71 & 1,65 & 1,61 & 1,56 \\
Wopt & 13,16 & 13,49 & 13,81 & 14,22 & 14,52 & 14,98 \\
IPI (opt) & 15,75 & 25,30 & 26,20 & 26,60 & 27,20 & 27,90 \\
$I_{C B R}$ après immersion & 4,18 & 68,00 & 79,00 & 81,00 & 95,00 & 111,00 \\
$I_{C B R} / I P I$ & 0,27 & 2,69 & 3,02 & 3,05 & 3,49 & 3,98 \\
\hline
\end{tabular}

(S)

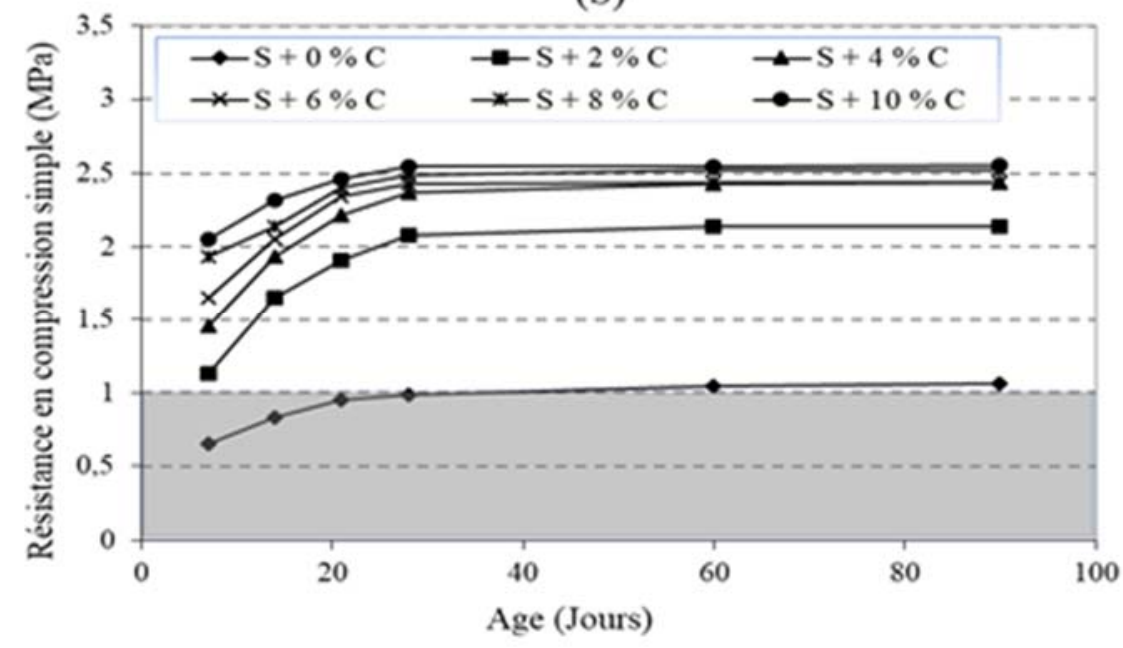

Figure 2. Evolution de la résistance à la compression simple $(\mathrm{Rc})$ des sédiments de la Soummam (S) traités avec différents dosages en ciment.

Les résistances en compression simple évoluent avec l'augmentation du taux de ciment comme le montre la figure 2 . Pour $2 \%$ de ciment l'augmentation est remarquable, l'effet du ciment est immédiat et les résistances ont doublé. A l'âge de 28 jours, les résistances obtenues tendent à se stabiliser pour toutes les formulations testées. 


\section{Thème 6 - Gestion durable des zones littorales et estuariennes}

La circulation des engins de chantier est autorisée lorsque le critère de traficabilité est satisfait. Le seuil $1 \mathrm{MPa}$ pour la résistance en compression simple $(\mathrm{Rc})$ est le minimum requis (LCPC et SETRA, 2000b). Cette condition est satisfaite dès 7 jours de cure. Ceci peut être expliqué par la création de cimentation (réaction pouzzolanique) provoquée par l'ajout de ciment induisant une cristallisation à long terme.

Dans une deuxième série d'essais, la granulométrie des sédiments a été modifiée à l'aide de fines de carrière dans des proportions allant de $5 \%$ à $25 \%$. L'impact recherché par ces ajouts est double : (i) une augmentation des résistances mécaniques par l'amélioration du squelette granulaire et une réduction du taux des matières organiques dans le mélange ; (ii) une valorisation de fines de carrière locales, résidus des stations de concassage de roches calcaires considérés comme des déchets.

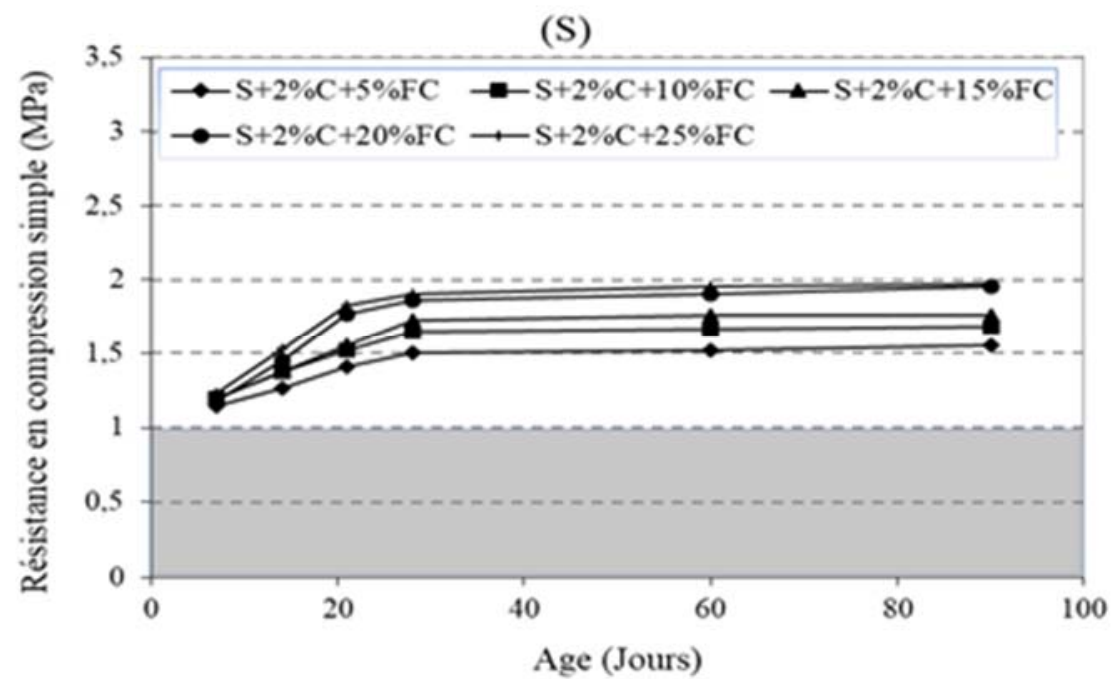

Figure 3. Evolution de la résistance à la compression simple $(R c)$ des sédiments $(S)$ traités avec différents dosages en fines de carrière.

Sur la figure 3, apparait une amélioration de Rc après ajout des fines de carrière. Les résistances sont supérieures au seuil de $1 \mathrm{MPa}$ requis dès le $7^{\mathrm{ème}}$ jour, approchant $2 \mathrm{MPa}$ à 28 jours. On peut conclure que l'effet des fines de carrière est stabilisateur. Ceci peut s'expliquer par sa granulométrie bien étalée et par sa finesse, ce qui améliore la cohésion au sein du sédiment. Toutefois, des résistances moindres sont obtenues pour les pourcentages élevés en fines de carrière. Cela peut être dû à l'excès de fines dans le produit final, tout en sachant que les fines de carrière sont constituées de $25 \%$ de particules de diamètre $<2 \mu \mathrm{m}$.

\section{Conclusion}

Nous avons exploré dans cette étude la possibilité de valoriser les sédiments étudiés dans le domaine routier, leurs principales caractéristiques ont été analysées. 


\section{XVI'̀mes Journées Nationales Génie Côtier - Génie Civil \\ Le Havre 2020}

Une prédominance du quartz et de la dolomite a été révélée lors de l'étude minéralogique. A travers les caractéristiques physiques mesurées, nous avons noté une prédominance de la portion des fines (plus de $80 \%$ de tamisât à $63 \mu \mathrm{m}$ ), un faible pourcentage d'argile et une présence non négligeable de matières organiques; ces dernières peuvent influencer négativement les comportements physique et mécanique du matériau.

D'après la classification selon le Guide technique (LCPC and SETRA 2000a) il s'agit de sols de classe $\mathrm{F}_{11} \mathrm{~A}_{2}$. Ceci favorise une utilisation en géotechnique, notamment avec le faible pourcentage d'argile présent dans les sédiments. Toutefois, les performances mécaniques obtenues montrent une portance en dessus des normes requises et des résistances faibles pour les matériaux routiers.

Une stabilisation de ces sédiments par le ciment a été réalisée en premier lieu afin d'obtenir les performances souhaitées. Puis un correcteur granulométrique représenté par une fine de carrière a été incorporé.

Le traitement au ciment a permet d'accroitre les performances mécaniques. L'indice portance immédiat IPI s'est nettement amélioré en fonction du dosage en ciment. Les rapports $\left(I_{C B R} / \mathrm{IPI}\right)$ sont tous nettement supérieurs à 1 . Ainsi, le risque de perte de cohésion en cas de saturation hydrique de la couche est écarté, ce qui est un critère de pérennité des traitements envisagés (LCPC et SETRA, 2000b).

Les résistances en compression simple $\mathrm{R}_{\mathrm{c}}$ obtenues ont augmenté comparées aux sédiments bruts, dépassant le seuil requis de $1 \mathrm{MPa}$ et permettant ainsi leur utilisation comme couche de forme de chaussée (LCPC et SETRA 2000b).

L'incorporation des fines de carrière en association avec $2 \%$ de ciment s'avère aussi efficace, les résistances en compression simple Rc sont satisfaisantes. Ces dernières sont proches de $2 \mathrm{MPa}$ à 28 jours et augmentent avec le taux de fines. Le critère de traficabilité est largement satisfait, l'utilisation de couches traitées de sédiments permet d'autoriser la circulation après quelques jours de mise en œuvre.

\section{Références bibliographiques}

AFNOR (1993a). Détermination des limites d'Atterberg-Limite de liquidité à la coupelleLimite de plasticité au rouleau (NF P94-051). Norme expérimentale, France, 15 p.

AFNOR (1993b). Préparation des matériaux traités aux liants hydrauliques ou non traités-Fabrication des éprouvettes de sables ou de sols fins par compression statique (NF P98-230-2). Norme expérimentale, France, 12 p.

AFNOR (1997). Indice CBR après immersion-Indice CBR immédiat-Indice portant immédiat (NF P94-078). Norme expérimentale, France, 12 p.

AFNOR (1999). Détermination des références de compactage d'un matériau-Essai Proctor normal-Essai Proctor modifié (NF P94-093). Norme expérimentale, France, 20 p.

ANGER B. (2014). Caractérisation de sédiments fins de retenues hydroélectriques en vue d'une orientation vers des filières de valorisation matière. Thèse de doctorat, Université de Caen, France, 316 p. 


\section{Thème 6 - Gestion durable des zones littorales et estuariennes}

BANOUNE B., MELBOUCI B., ROSQUOËT F., LANGLET T. (2016). Treatment of river sediments by hydraulic binders for valorization in road construction. Bull Eng Geol Environ. Vol. 75(4), pp 1505-1517. https://doi.org/10.1007/s10064-015-0844-4

BOUTOUIL M. (1998). Traitement des vases de dragage par solidification / stabilisation à base de ciment et additifs. Thèse de doctorat, Université du Havre, France, 197 p.

DUBOIS V., ABRIAK N.E., ZENTAR R., BALLIVY G. (2009). The use of marine sediments as a pavement base material. Waste Management, Vol. 29, pp 774-782. https://doi.org/10.1016/j.wasman.2008.05.004

LCPC, SETRA (2000a). Réalisation des remblais et des couches de forme, guide technique. Fascicule II-Annexes techniques, 2ème édition, Paris, 211 p.

LCPC, SETRA (2000b). Traitement des sols à la chaux et/ou aux liants hydrauliques. Application à la réalisation des remblais et des couches de forme, guide technique, Paris, $246 \mathrm{p}$.

LEVACHER D., COLIN D., CARINA A., DUAN Z., SUN L. (2006). Recyclage et valorisation de sédiments fins de dragage à usage de matériaux routiers. IXème journées nationales Génie Civil- Génie Côtier, Brest, pp 603-612. https://doi.org/10.5150/jngcgc.2006.058-L MAHERZI W., BEN ABDELGHANI F. (2014). Dredged marine raw sediments geotechnical characterization for their reuse in road construction. Engineering Journal, Vol. 18(4), pp 27-37. https://doi.org/10.4186/ej.2014.18.4.27

MAROUF H. (2018). Valorisation des sédiments issus du dragage du barrage de Bouhanifia et du port d'Oran, Thèse de Doctorat, Université de Mostaganem, Algérie, $124 \mathrm{p}$.

REKIK B., BOUTOUIL M. (2011). Geotechnical properties of dredged marine sediments treated at high water/cement ratio. Geo-Marine Letters, Vol. 29, pp 171-179. https://doi.org/10.1007/s00367-009-0134-x

SCORDIA P. (2008). Caractérisation et valorisation de sédiments fluviaux pollués et traités dans les matériaux routiers. Thèse de Doctorat, Ecole centrale de Lille, 202 p.

SEMCHA A. (2006). Valorisation des sédiments de dragage : Applications dans le BTP, cas du barrage de Fergoug. Thèse de Doctorat, U. de Reims, France, 175 p.

SILITONGA E. (2010). Valorisation des sédiments marins contaminés par solidification/stabilisation à base de liants hydrauliques et de fumée de silice. Thèse de Doctorat, Université de Caen, 245 p.

WANG D. (2011). Solidification et valorisation de sédiments du port de Dunkerque en travaux routiers. Thèse de Doctorat, Ecole des Mines de Douai et Université Lille 1, France, $174 \mathrm{p}$.

ZENTAR R., ABRIAK N.E, TRAN N. (2009). Caractérisation et étude de l'impact sur l'environnement de sédiments dragués dans le nord de France. Revue Paralia, Vol. 2, pp 4.1 - 4.12. https://doi.org/10.5150/revue-paralia.2009.004 